\title{
Numerical simulation of continuous damage and fracture in metal-forming processes with 3D mesh adaptive methodology
}

\author{
F. Yang ${ }^{1}$, A. Rassineux ${ }^{1}$, C. Labergere $^{2}$, K. Saanouni ${ }^{2}$ \\ ${ }^{1}$ Sorbonne Université, Université de Technologie de Compiègne, UMR CNRS/UTC 7337, fangtao.yang@utc.fr \\ ${ }^{2}$ Université de Technologie de Troyes, ICD/LASMIS, UMR STMR 6279, carl.labergere@utt.fr
}

\begin{abstract}
An h-adaptive remeshing scheme dedicated to the simulation of cracked structures due to the ductile damage such as metal forming processes is proposed. Cracks are represented using a procedure based on fully damaged elements deletion. Element size inside the domain and along the crack path, is driven by error indicators based on geometrical considerations and the derivatives of physical quantities calculated by Diffuse Approximation and specific fields transfer procedures and an automatic adaptation of the time loading sequences are proposed. Emphasis is put on the remeshing issues.
\end{abstract}

Key words - Adaptivity, Error estimation, Forming processes.

\section{Instruction}

When a metallic part is formed by large plastic (or viscoplastic) strains, ductile damage is expected to occur inside zones where the plastic flow is highly localized. When ductile damage occurs, the propagation of macroscopic cracks induces severe changes of topology and frequent remeshing must be performed in order to avoid large mesh distortion or element entanglement encountered in a Lagrangian formulation. As our work is focused on meshing and remeshing aspects, we have concentrated our classification of techniques on this topic. In this context, a number of methods have been proposed to represent ductile cracks occurring inside zones where the plastic flow localizes mostly based on nodal relaxation and cohesive elements [1], element deletion procedures, associated with a critical damage criterion [2][3], enrichment techniques in order to represent with accuracy the fields at the crack tip may be obtained by a mesh refinement with specific patterns or by the use of extended finite elements. Among the last class of techniques, the extended finite element method (XFEM) based on the addition of an enriched basis into the existing finite element mesh is now widely used [4] to avoid frequent remeshing and to cope with the problem of mesh size dependency which alters crack path prediction. The present work deals with the prediction of ductile fracture in metal forming where large inelastic (plastic or viscoplastic) strains take place using a fully adaptive scheme combining h-adaptivity (refinement and coarsening), error indicators, field transfer operators and an adaptive loading sequences technique. Various methods [5] mostly in 2D and based on finite element have been proposed to predict the ductile fracture occurrence inside metallic parts formed by large plastic deformation based on damage-induced loss of stiffness together with mesh adaptation.

\section{Thermo-elasto-viscoplastic constitutive equations}

The model and its numerical implementation has been presented in reference [2]. The fully coupled thermomechanical constitutive equations have been implemented into ABAQUS/Explicit FE code using the user defined subroutine VUMAT. The dynamic explicit resolution procedure has been used in order to solve the thermomechanical evolution problem based on the two weak forms relative to the equilibrium and thermal equations. The local integration of the fully coupled constitutive equations is performed using an iterative implicit scheme based on the well-known elastic prediction - plastic correction radial return scheme applied to a reduced number of equations. 


\section{3D-adaptive numerical simulation methodology}

The FEM based numerical simulation of forming processes involving large inelastic (plastic or viscoplastic) deformations requires adequate spatial discretization of the deformed parts. Indeed, during Lagrangian-based numerical simulations of forming processes, frequent remeshing is needed during the computation in order to avoid large mesh distortion and also to control the errors caused by the approximation of the thermo-mechanical fields. The size of the mesh is then driven by appropriate error indicators based both on physical gradient fields (stress, plastic strain, temperature, damage ....) and also on the curvature of the external boundary of the domain allowing a better control of the contact interface. Procedures to compute the curvature of boundary surface on the mesh by diffuse approximation [6] have been used. The efficiency of the adaptive methodology is based on a discretization of the parts using the h-method into tetrahedral elements. To achieve this goal, the following methodology is carried out :

- FEM software ABAQUS/EXPLICIT is used to solve the initial and boundary value problem. At each integration point, the local constitutive equations are introduced using a VUMAT subroutine.

- Geometrical and physical error indicators are used to determine an "optimal" size map of the mesh.

- A local 3D tetrahedra remesher is used to perform a local remeshing in "active" areas.

A Local remeshing instead of a complete remeshing of the whole structure is a crucial issue of this work. Associated to a local remeshing, a local transfer of variables is performed. The thermomechanical fields are transferred from the old to the new mesh based on finite element approximation for the nodal variables and on appropriate diffuse interpolation of the state variables defined at integration points [2].A procedure for adjusting the next loading sequence according to the size and number of fully damaged elements if any is proposed.

\subsection{Diffuse approximation issues}

A moving least square approximation denoted as Diffuse Approximation [3] is used in its interpolating form to calculate derivatives of quantities which are used to build error indicators and after remeshing to recover the various thermomechanical fields " $S_{\text {phys }}$ " (plastic strain, stress, damage, isotropic hardening,... ) located at the integration points of each element. Our error indicators are based on 2nd order derivatives while a first order interpolation is used for the field transfer procedure. In the vicinity of an evaluation point $M=(x, y, z)^{T}$ each scalar field " $S_{\text {phys }}$ " is considered as a Taylor expansion where $\widetilde{M}=(\widetilde{x}, \widetilde{y}, \widetilde{z})^{T}$ is expressed in the global system of coordinates. The vector of coefficients $\{\alpha(M)\}$ varies with the position of the evaluation point as shown in the equation (1).

$$
\begin{aligned}
S(\widetilde{x}, \widetilde{y}, \widetilde{z})= & \left\langle 1, \widetilde{x}-x, \widetilde{y}-y, \widetilde{z}-z,(\widetilde{x}-x)^{2},(\widetilde{y}-y)^{2},(\widetilde{z}-z)^{2},\right. \\
& (\widetilde{x}-x)(\widetilde{y}-y),(\widetilde{x}-x)(\widetilde{z}-z),(\widetilde{y}-y)(\widetilde{z}-z)\rangle\{\alpha(M)\} \\
= & \langle P(\widetilde{M}-M)\rangle\{\alpha(M)\}
\end{aligned}
$$

Thereafter, an error indicator based on the Hessian of the plastic dissipation is proposed [2]. In addition, the size of the mesh is also driven by an empirical functions based on the assumption that the behavior of the plastically deformed part may be decomposed in a number of thermomechanical states : elastic, plastic homogeneous with low damage value, highly localized plastic zone with moderate damage, plastic zone with high damage value.

\subsection{Mesh adaptation}

In this paper, we shall only focuss on the refinement of the existing mesh. Mesh coarsening is a difficult task, however essential, will not be addressed here. During the simulation, frequent 3D remeshing may be carried out and it is therefore essential to use a robust and efficient technique to refine the mesh. The method has been proposed in [7] but has never been used in this context.

To validate the efficiency of the local remeshing technique, we propose an geometrical validation of the remeshing tools. An initial mesh of a sphere is shown in figure 1. When A thin cutting plane is 


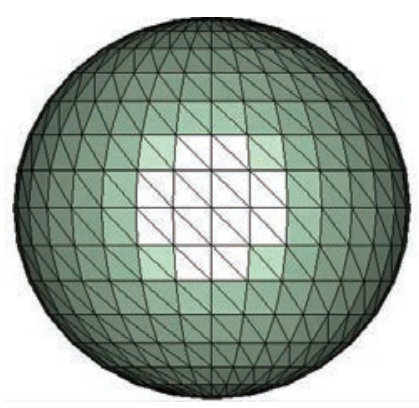

FIGURE 1 - Initial mesh

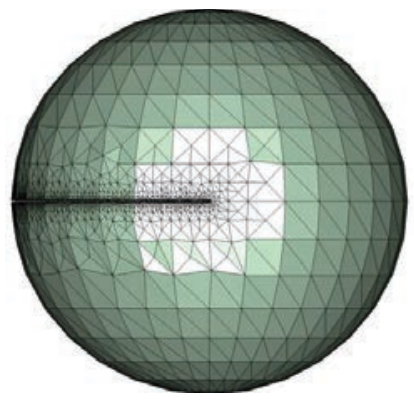

FIGURE 2 - Adapted mesh

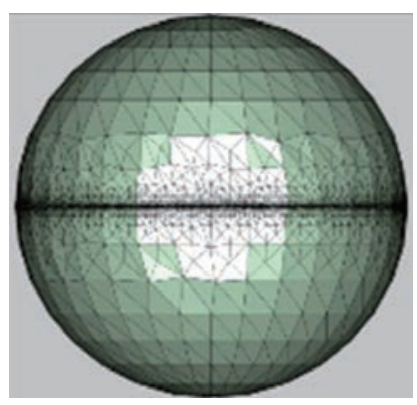

FIGURE 3 - Final mesh

moved step by step cutting the sphere from its equator, the mesh is refined with respect to this analytical simulated crack as shown in figure 2. Finally, when the cutting plane cut through the whole equator plane of the sphere, the sphere is divided into two parts by the analytical simulated crack as shown in figure 3 . The mesh within the zones which are near the both surfaces (top and down) of the crack are well refined. Figure 3 shows a close up of the mesh refinement along the crack.

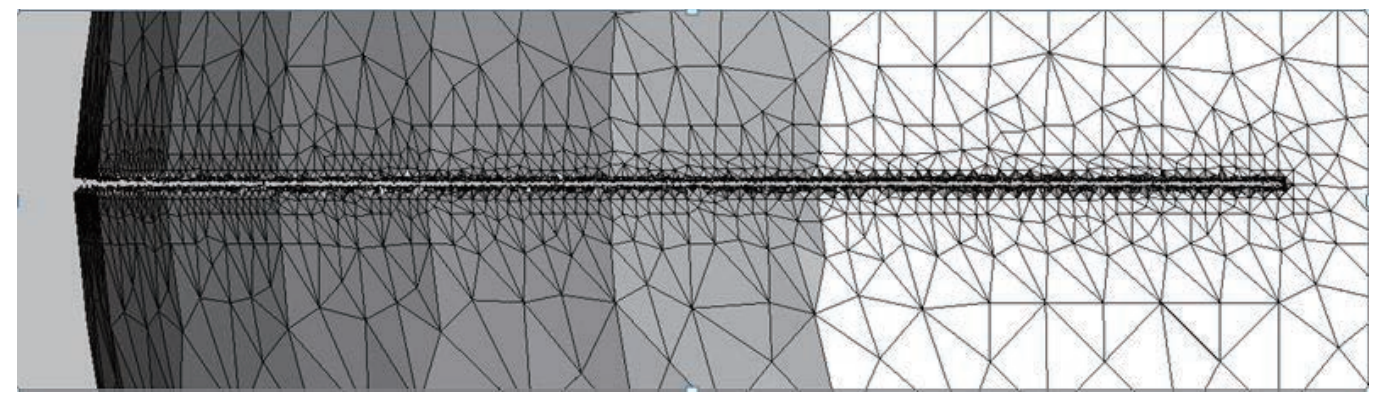

FIGURE 4 - A more clear view of the refinement of the mesh

\section{Reference}

[1] Bouchard, Pierre-Olivier and Bay, François and Chastel, Yvan and Tovena, Isabelle. Crack propagation modelling using an advanced remeshing technique, Computer methods in applied mechanics and engineering, Elsevier, 723-742, 2000.

[2] Labergere, Carl and Rassineux, Alain and Saanouni, Khemais. Numerical simulation of continuous damage and fracture in metal-forming processes with 2D mesh adaptive methodology, Finite Elements in Analysis and Design, Elsevier, 46-61, 2014.

[3] Borouchaki, Houman and Laug, Patrick and Cherouat, Abel and Saanouni, Khémaïs. Adaptive remeshing in large plastic strain with damage, International journal for numerical methods in engineering, Wiley Online Library, 1-36, 2005.

[4] Moës, Nicolas and Belytschko, Ted. Extended finite element method for cohesive crack growth, International journal for numerical methods in engineering, Elsevier, 813-833, 2002.

[5] Borouchaki, Houman and Laug, Patrick and Cherouat, Abel and Saanouni, Khémaïs. Aspects of ductile fracture and adaptive mesh refinement in damaged elasto-plastic materials, International journal for numerical methods in engineering, Wiley Online Library, 29-54, 2001.

[6] Rassineux, Alain and Villon, Pierre and Savignat, J-M and Stab, Olivier. Surface remeshing by local Hermite diffuse interpolation, International Journal for Numerical Methods in Engineering, Wiley Online Library, 3149, 2000.

[7] Rassineux, A and Breitkopf, P and Villon, P. Simultaneous surface and tetrahedron mesh adaptation using mesh-free techniques, International Journal for Numerical Methods in Engineering, Wiley Online Library, 371-389, 2003. 